\title{
Homological mirror symmetry is T-duality for $\mathbb{P}^{n}$
}

\author{
BOHAN FANG
}

\begin{abstract}
In this paper, we apply the idea of T-duality to projective spaces. From a connection on a line bundle on $\mathbb{P}^{n}$, a Lagrangian in the mirror Landau-Ginzburg model is constructed. Under this correspondence, the full strong exceptional collection $\mathcal{O}_{\mathbb{P} n}(-n-1), \ldots$, $\mathcal{O}_{\mathbb{P}^{n}}(-1)$ is mapped to standard Lagrangians in the sense of [23]. Passing to constructible sheaves, we explicitly compute the quiver structure of these Lagrangians, and find that they match the quiver structure of this exceptional collection of $\mathbb{P}^{n}$. In this way, T-duality provides quasi-equivalence of the Fukaya category generated by these Lagrangians and the category of coherent sheaves on $\mathbb{P}^{n}$, which is a kind of homological mirror symmetry.
\end{abstract}

\section{Introduction}

Mirror symmetry was first observed for Calabi-Yau manifolds. It has been extended to Fano cases by considering Landau-Ginzburg models as mirrors to Fano varieties [16]. A Landau-Ginzburg model is a non-compact manifold equipped with a complex-valued holomorphic function called the superpotential. In this paper, we study the approach of homological mirror symmetry proposed by Kontsevich [19]. Kontsevich suggests to investigate homological mirror symmetry as the equivalence of the Fukaya category on the A-model side and the category of coherent sheaves on its mirror Bmodel for Calabi-Yau manifolds. Kontsevich [18] and Hori-Iqbal-Vafa [15] discuss homological mirror symmetry in the case of Fano manifolds. Following this line, Auroux et al. [5] prove homological mirror symmetry for weighted projective planes (and their non-commutative deformations) and Del Pezzo surfaces [6]. Abouzaid [1,2] proves the case of all smooth projective toric varieties using tropical geometry. Bondal and Ruan also announce a result for weighted projective spaces [7].

Strominger et al. [24] conjecture that mirror symmetry is a manifestation of T-duality on a special Lagrangian torus fibration. In the case of toric Fano varieties $[14,20]$, the moment map produces a fibration by Lagrangian tori. Auroux [4] discusses the relation between the SYZ conjecture and Fano varieties. Recently, Chan and Leung [9] apply T-duality with quantum 
corrections to recover mirror symmetry for toric Fano varieties. This paper deals with homological mirror symmetry for the projective space $\mathbb{P}^{n}$, using the philosophy of T-duality. We will apply this T-duality to any holomorphic line bundle over $\mathbb{P}^{n}$ and obtain a Lagrangian in the Landau-Ginzburg mirror. We define $n+1$ Lagrangians $\mathcal{L}(-1), \ldots, \mathcal{L}(-n-1)$ in the mirror which arise via T-duality of the collection $\mathcal{O}(-1), \ldots, \mathcal{O}(-n-1)$. We roughly state our theorem here.

Theorem 1.1. The derived Fukaya category containing $\mathcal{L}(-1), \ldots$, $\mathcal{L}(-n-1)$ is equivalent to the derived category of coherent sheaves on $\mathbb{P}^{n}$.

The definition of this Fukaya category will be specified later. We remark that the Lagrangians we are considering are submanifolds in $\left(\mathbb{C}^{*}\right)^{n}$ considered as the cotangent bundle of $\left(S^{1}\right)^{n}$. While $\left(\mathbb{C}^{*}\right)^{n}$ together with a certain superpotential $W$ is the Landau-Ginzburg mirror of $\mathbb{P}^{n}$ in the sense of $[15,18]$, we do not explicitly consider the superpotential $W$ here. This differs from the case in $[5,6]$, in which the authors consider the Fukaya-Seidel category consisting of vanishing cycles in a generic fiber of the superpotential. However, the Lagrangians in this paper are somehow like the vanishing thimbles. Under some tentative calculation, the images of the Lagrangiang $\mathcal{L}(k)$ under the superpotential $W$ are not horizontal half-lines going from critical values to the positive infinity. Instead, they are "thickened" rays. Thus the Fukaya category we are using differs from the original idea of [15]. We hope a renormalization process suggested by Auroux [4] and Hori and Vafa [16] will remedy this problem in the future.

Remark 1.2. When computing this Fukaya category, we pass to the dg category of constructible sheaves by the results of $[22,23]$. The constructible sheaves coming from $\mathcal{L}(-1), \ldots, \mathcal{L}(-n-1)$ are constructible with respect to a particular stratification. This stratification coincides with Bondal $[7,8]$ in the case of projective spaces. The situation in more general cases and the comparison with Bondal's results will be discussed in [11].

The use of an explicit exceptional collection of $\mathbb{P}^{n}$ is basically for convenience only. T-duality produces an object for any line bundle, as does the unique functor defined to agree with T-duality on a generating exceptional collection. The maps of objects do not a priori coincide. This issue is addressed in [11], and they turn out to be the same. The method of this paper is extended to treat all projective toric varieties in [11], thus recovering Abouzaid's result [2]. 


\section{Acknowledgments}

I would like to thank my advisor, Eric Zaslow, for showing me the idea of T-duality on projective spaces and for valuable discussions and encouragement throughout this project. I would also like to thank Chiu-Chu Liu and David Treumann for the discussions when writing a joint paper [11], which lead to important improvement of this one.

\section{Mirror of projective spaces}

In this section, we describe the mirror of the projective space $\mathbb{P}^{n}$ over $\mathbb{C}$. Roughly speaking, the mirror of $\mathbb{P}^{n}$ is a Landau-Ginzburg model, i.e., a noncompact manifold with a superpotential [16,18]. The SYZ conjecture [24] suggests to construct the mirror of $\mathbb{P}^{n}$ via T-duality on the torus fibration over the moment polytope, as argued in [20]. The superpotential is conjecturally given by Fukaya-Oh-Ohta-Ono's $m_{0}$ obstruction [12]. This has been investigated by Cho and $\mathrm{Oh}[10]$. Following this idea, we give a description of the mirror of $\mathbb{P}^{n}$ as a complexified moduli space of special Lagrangians. The material in this section is from [4].

On $\mathbb{P}^{n}$ with its Fubini-Study metric and corresponding symplectic form $\omega$, the torus $T^{n}$ acts via

$$
\left(\theta_{1}, \cdots, \theta_{n}\right) \cdot\left(z_{0}: z_{1}: \cdots: z_{n}\right)=\left(z_{0}: e^{2 \pi i \theta_{1}} z_{1}: e^{2 \pi i \theta_{2}} z_{2}: \cdots: e^{2 \pi i \theta_{n}} z_{n}\right)
$$

This action produces a moment map $\phi: \mathbb{P}^{n} \rightarrow \Delta$ given by

$$
\left(z_{0}: z_{1}: \cdots: z_{n}\right) \mapsto\left(\frac{\left|z_{1}\right|^{2}}{\sum_{i=0}^{n}\left|z_{i}\right|^{2}}, \frac{\left|z_{2}\right|^{2}}{\sum_{i=0}^{n}\left|z_{n}\right|^{2}}, \ldots, \frac{\left|z_{n}\right|^{2}}{\sum_{i=0}^{n}\left|z_{i}\right|^{2}}\right),
$$

where

$$
\Delta=\left\{\left(x_{1}, x_{2}, \ldots, x_{n}\right): x_{i} \geq 0, \sum_{i=1}^{n} x_{i} \leq 1\right\}
$$

This moment map is a torus fibration outside of the boundary.

Let $D$ be the boundary divisor of $\phi$, i.e., by $D=\phi^{-1}(\partial \Delta)=\left\{\left(z_{0}\right.\right.$ : $\left.\left.z_{1}: \cdots: z_{n}\right) \in \mathbb{P}^{n} \mid z_{0}, \ldots, z_{n}=0\right\}$. The holomorphic $n$-form $\Omega$ on $\mathbb{P}^{n} \backslash D$ is $d \log z_{1} \wedge \cdots \wedge d \log z_{n}$ in coordinates $\left(1: z_{1}: \cdots: z_{n}\right) . \quad$ By a special Lagrangian we mean a Lagrangian submanifold $L$ with a constant phase $\vartheta$, i.e., $\left.\operatorname{Im}\left(e^{-i \vartheta} \Omega\right)\right|_{L}=0$. It is easy to see that the moment map $\phi$ defines 
a $T^{n}$-orbit fibration on $\mathbb{P}^{n} \backslash D$. Because of the following lemma, this is a special Lagrangian fibration.

Proposition 2.1. The $T^{n}$-orbits in $\mathbb{P}^{n} \backslash D$ are special Lagrangians.

Proof. It is a classical fact that any $T^{n}$-orbit is a Lagrangian. Notice the holomorphic form $\Omega$ on $\mathbb{P}^{n} \backslash D$ is $d \log z_{1} \wedge \cdots \wedge d \log z_{n}$. We see the restriction of $\Omega$ on the orbit gives rise to phase $n \pi / 2$.

On $\mathbb{P}^{n}$, define a polar coordinate system $(r, \theta)$ by $z_{k}=r_{k} e^{i \theta_{k}}$ at the point $\left(1: z_{1}: \cdots: z_{n}\right)$. The coordinate $r$ is the coordinate on the base of the fibration $\phi$, while $\theta$ lives in the fiber.

The mirror $M$ is constructed as the moduli space of the torus fibers together with flat connections on them.

Definition 2.2. The complexified moduli space $M$ consists of pairs $(L, \nabla)$. Here $L$ is a $T^{n}$-orbit in $\mathbb{P}^{n} \backslash D$, and $\nabla$ is a flat $U(1)$ connection on the trivial line bundle over $L$ up to gauge equivalence.

We omit the details of the complex and the symplectic structures on $M$. The result is stated here without any explanation. Any $T^{n}$-orbit in $\mathbb{P}^{n} \backslash D$ has to be a fiber of $\phi$. We write a fiber

$$
L(r):=S^{1}\left(r_{1}\right) \times \cdots \times S^{1}\left(r_{n}\right)=\left\{\left(1: z_{1}: \cdots: z_{n}\right),\left|z_{i}\right|=r_{i}\right\}
$$

for $r=\left(r_{1}, \ldots, r_{n}\right) \in\left(\mathbb{R}^{+}\right)^{n}$. It is obvious that $L\left(r_{1}, \ldots, r_{n}\right)$ is mapped to a point in $\Delta$ by the moment map $\phi$. The flat connection $\nabla$ on $L$ can be represented as $\sum \gamma_{i} d \theta_{i}$. Therefore we can give a coordinate system to $M$ by $(r, \gamma)$, where $r=\left(r_{1}, \ldots, r_{n}\right)$ and $\gamma=\left(\gamma_{1}, \ldots, \gamma_{n}\right)$. Notice $\gamma_{i}$ takes value in $S^{1}=\mathbb{R} / \mathbb{Z}$. Since $\mathbb{P}^{n} \backslash D$ and $M$ are dual fibrations on the same base, they share the same coordinate $r$ on the base. With these coordinates $(r, \gamma)$ in hand, the symplectic structure on $M$ is defined as following.

Proposition 2.3. The symplectic structure on $M$ is given by

$$
\omega^{\vee}=(2 \pi)^{n} \sum d \log r_{i} \wedge d \gamma_{i}
$$

Let $y_{i}=\log r_{i}$, and we see that $\omega^{\vee}=(2 \pi)^{n} \sum d y_{i} \wedge d \gamma_{i}$. Hence $M$ carries the symplectic structure of the cotangent bundle over $\left(S^{1}\right)^{n}$. The coordinates $\gamma_{i}$ are on the base $\left(S^{1}\right)^{n}$ while $y_{i}$ live on the fiber. The coordinate systems $(r, \gamma)$ and $(y, \gamma)$ are used throughout the paper with the relation $y_{i}=\log r_{i}$. We sometimes write $T^{*}\left(S^{1}\right)^{n}$ as a synonym for the moduli space 
$M$, since we primarily consider the symplectic structure of $M$. For the complex structure on $M$, there is

Proposition 2.4. The moduli space $M$ is biholomorphic to a subset of $\left(\mathbb{C}^{*}\right)^{n}$, given by the complex coordinates $z_{j}=\exp \left(-2 \pi \phi_{j}(L)\right) \operatorname{hol}_{\nabla}\left(\left[S^{1}\left(r_{j}\right)\right]\right)$. The map $\phi_{j}$ is the $j$-th component of the moment map $\phi$, while hol $\operatorname{lo}_{\nabla}\left(\left[S^{1}\left(r_{j}\right)\right]\right)$ is the holonomy of $\nabla$ with respect to $\left[S^{1}\left(r_{j}\right)\right]$.

A straightforward calculation shows that

$$
z_{j}(L, \nabla)=\exp \left(-\frac{2 \pi r_{j}^{2}}{1+\sum_{i=1}^{n} r_{i}^{2}}+2 \pi i \gamma_{j}\right)
$$

It can be verified that $\omega^{\vee}$ is indeed a Kähler structure with respect to the complex structure.

The superpotential of $M$ is obtained by $m_{0}$ obstruction to Floer homology, roughly speaking, counting holomorphic discs attached to a special Lagrangian. For here, it is explicitly given by

Proposition 2.5. The superpotential $W$ on $M$ is given by

$$
W=z_{1}+\cdots+z_{n}+\frac{e^{-2 \pi}}{z_{1} z_{2} \cdots z_{n}} .
$$

\section{T-duality and constructible sheaves}

\subsection{T-duality on torus fibers}

As the definition of $M$ shows, we can go from a flat $U(1)$ gauge field on a special Lagrangian fiber in $\mathbb{P}^{n} \backslash D$ to a point in the corresponding dual fiber in $M$. Leung-Yau-Zaslow and Arinkin-Polishchuk apply a similar transformation in [21] and [3], respectively, and go from a Lagrangian to a gauge field. Here we do this in the other way, namely, from a gauge field to a Lagrangian. From any holomorphic line bundle on $\mathbb{P}^{n}$, we construct an exact Lagrangian in $M$.

We endow a line bundle $E$ on $\mathbb{P}^{n}$ with a $T^{n}$-invariant hermitian metric $h$, constant on each fiber of $\phi$. The canonical connection on $E$ with respect to this metric is $\nabla_{E, h}=d-i A_{E, h}$, with the connection 1-form $A_{E, h}=i \partial h$. $h^{-1}$ in some trivialization. ${ }^{1}$ The restriction of $\nabla_{E, h}$ to any fiber $L$ of $\left.\phi\right|_{\mathbb{P}^{n} \backslash D}$ gives rise to a connection $\left.\nabla_{E, h}\right|_{L}$ on the special Lagrangian $L$.

\footnotetext{
${ }^{1}$ We use the physics notation, such that $A_{E, h}$ is real-valued.
} 
Lemma 3.1. $\left.\nabla_{E, h}\right|_{L}$ is a flat connection over $L$.

Proof. The connection $\nabla_{E, h}$ can be written as $d-i A_{E, h}$. The connection 1-form $A_{E, h}$ is given by

$$
\begin{aligned}
A_{E, h} & =i \partial h \cdot h^{-1} \\
& =-h^{-1} \cdot \sum_{i=1}^{n} \frac{\partial h}{\partial r_{i}} r_{i} d \theta_{i}+\text { terms in } d r_{1}, \ldots, d r_{n}
\end{aligned}
$$

The metric $h$ is a function of $r=\left(r_{1}, \ldots, r_{n}\right)$ and it does not depend on $\theta$, since it is constant on each fiber. Hence the restriction of $A_{E, h}$ on each fiber gives vanishing curvature.

Recall from Section 1 that $M$ is the space of non-singular $T^{n}$-orbits together with flat connections, and $M \cong\left(\mathbb{C}^{*}\right)^{n}$ as a symplectic manifold with coordinates $(r, \gamma)$ and the symplectic form

$$
\omega^{\vee}=(2 \pi)^{n} \sum_{i=1}^{n} d \log \left(r_{i}\right) \wedge d \gamma_{i}
$$

We define the submanifold $\mathcal{L}(E, h) \subset M$ to be

$$
\left\{\left(L,\left.\nabla_{E, h}\right|_{L}\right): L \text { is an } T^{n} \text {-orbit in } \mathbb{P}^{n} \backslash D\right\}
$$

In coordinates, we see that $\left.\nabla_{E, h}\right|_{L}$ is smooth as a function of the fiber $L$, and hence $\mathcal{L}(E, h)$ is a submanifold of $M$.

Proposition 3.2. $\mathcal{L}(E, h)$ is an exact Lagrangian submanifold.

Proof. In the coordinates $(r, \gamma)$ of $M$,

$$
\begin{aligned}
\mathcal{L}(E, h)=\{ & \left(r_{1}, \ldots, r_{n} ;-h^{-1} \cdot \frac{\partial h}{\partial r_{1}} r_{1}, \ldots,-h^{-1} \cdot \frac{\partial h}{\partial r_{n}} r_{n}\right), \\
& \left.\left(r_{1}, \ldots, r_{n}\right) \in\left(\mathbb{R}^{+}\right)^{n}\right\}
\end{aligned}
$$


The tangent space of $\mathcal{L}(k, h)$ at any point is spanned by the collection $\left\{\partial_{r_{i}}-\right.$ $\left.h^{-1} \cdot \sum_{j=1}^{n} r_{j} \frac{\partial^{2} h_{j}}{\partial r_{i} \partial r_{j}} \partial_{\gamma_{j}}, 1 \leq i \leq n\right\}$. We have

$$
\begin{aligned}
& \omega^{\vee}\left(\partial_{r_{i}}-h^{-1} \cdot \sum_{j=1}^{n} r_{j} \frac{\partial^{2} h_{j}}{\partial r_{i} \partial r_{j}} \partial_{\gamma_{j}}, \partial_{r_{i^{\prime}}}-h^{-1} \cdot \sum_{j^{\prime}=1}^{n} r_{j^{\prime}} \frac{\partial^{2} h_{j^{\prime}}}{\partial r_{i^{\prime}} \partial r_{j^{\prime}}} \partial_{\gamma_{j^{\prime}}}\right) \\
& \quad=(2 \pi)^{n} h^{-1}\left(-\frac{\partial^{2} h_{j}}{\partial r_{i} \partial r_{i^{\prime}}}+\frac{\partial^{2} h_{j}}{\partial r_{i} \partial r_{i^{\prime}}}\right) \\
& \quad=0 .
\end{aligned}
$$

The simple-connectedness of $\mathcal{L}(E, h)$ implies it is exact.

\subsection{Objects in the Fukaya category}

In this subsection, we show that for the canonical metric $h_{k}$ on $\mathcal{O}(k)$, the Lagrangian $\mathcal{L}\left(\mathcal{O}(k), h_{k}\right)$ can be endowed with a canonical brane structure, thus it is an object in the Fukaya category $\operatorname{Fuk}(M)$.

For the line bundle $\mathcal{O}(1)$, on the open set $U=\left\{\left(1: z_{1}: \cdots: z_{n}\right)\right\} \subset \mathbb{P}^{n}$ we can write any $\left.x \in \mathcal{O}(1)\right|_{U}$ as $\left\{\left(1: z_{1}: \cdots: z_{n}\right), \xi\right\}$ by a local trivialization of $\mathcal{O}(1)$. The natural $T^{n}$-invariant hermitian metric $h_{1}$ on $\mathcal{O}(1)$ is given by

$$
h_{1}(x, y)=\frac{\langle\xi, \eta\rangle}{1+\sum_{i=1}^{n}\left|z_{i}\right|^{2}} .
$$

Writing $z_{i}=r_{i} e^{i \theta_{i}}$, the canonical connection $\nabla_{\mathcal{O}(1), h_{1}}=d-i A_{\mathcal{O}(1), h_{1}}$ on $\mathcal{O}(1)$ with respect to $h_{1}$ is

$$
\begin{aligned}
A_{\mathcal{O}(1), h_{1}} & =i \partial h_{1} \cdot h_{1}^{-1} \\
& =\frac{r_{1}^{2} d \theta_{1}}{1+\sum_{i=1}^{n} r_{i}^{2}}+\cdots+\frac{r_{n}^{2} d \theta_{n}}{1+\sum_{i=1}^{n} r_{i}^{2}}+\text { terms of } d r_{1}, \ldots, d r_{n} .
\end{aligned}
$$

Hence, the Lagrangian

$$
\begin{aligned}
& \mathcal{L}\left(\mathcal{O}(1), h_{1}\right)=\{(\left(r, \gamma^{(1)}(r)\right): r=\left(r_{1}, \ldots, r_{n}\right) \in\left(\mathbb{R}^{+}\right)^{n}, \\
&\left.\gamma^{(1)}(r)=\left(\frac{r_{1}^{2}}{1+\sum_{i=1}^{n} r_{i}^{2}}, \ldots, \frac{r_{n}^{2}}{1+\sum_{i=1}^{n} r_{i}^{2}}\right)\right\},
\end{aligned}
$$

which is obviously the graph of the $\left(S^{1}\right)^{n}$-valued function $\gamma^{(1)}$. For any other holomorphic line bundle $\mathcal{O}(k)$, let $h_{k}$ denote $\left(h_{1}\right)^{k}$, and this construction gives rise to a Lagrangian (Figure 1). 

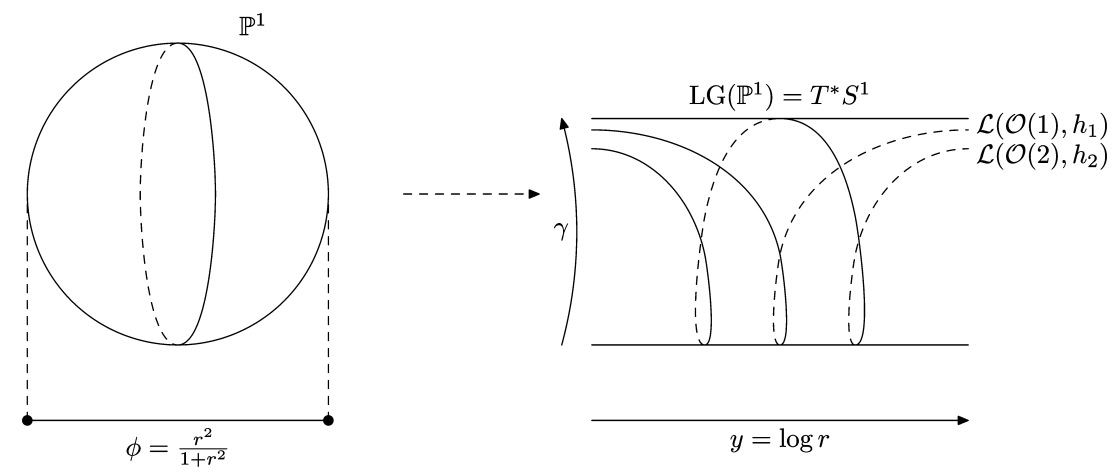

Figure 1: Fiberwise T-duality transformation for $\mathbb{P}^{1}$. The Lagrangians $\mathcal{L}\left(\mathcal{O}(1), h_{1}\right)$ and $\mathcal{L}\left(\mathcal{O}(2), h_{2}\right)$ shown are obtained from line bundles $\mathcal{O}(1)$ and $\mathcal{O}(2)$, respectively.

$$
\mathcal{L}\left(\mathcal{O}(k), h_{k}\right)=\left\{\left(r, \gamma^{(k)}\right): r=\left(r_{1}, \ldots, r_{n}\right) \in\left(\mathbb{R}^{+}\right)^{n}, \gamma^{(k)}=k \gamma^{(1)}\right\}
$$

We adapt the definition of the Fukaya category $\operatorname{Fuk}(M)$ for cotangent bundles from [23]. The moduli space $M$ is symplectomorphic to the cotangent bundle $M=T^{*}\left(S^{1}\right)^{n}$, where $S^{1}=\mathbb{R} / \mathbb{Z}$. Moreover, it is already equipped with a standard symplectic form $\omega^{\vee}=(2 \pi)^{n} \sum d y_{j} \wedge d \gamma_{j}$. The Fukaya category of a cotangent bundle has been defined in [23], ${ }^{2}$ and we apply that definition to $M=T^{*}\left(S^{1}\right)^{n}$. The variable $\gamma_{j}$ are the coordinates on the base which are periodic with period 1 and $y_{j}$ are the coordinates on the fiber. Fix a metric on the base $\left(S^{1}\right)^{n}$ to be $g=d \gamma_{1}^{2}+d \gamma_{2}^{2}+\cdots+d \gamma_{n}^{2}$. We would like to employ the result of [23] to perform calculations in the Fukaya category. The projective space $\mathbb{P}^{n}$ has an exceptional collection. We choose one here: $\mathcal{O}(-n-1), \mathcal{O}(-n), \ldots, \mathcal{O}(-1)$. The philosophy is to show that the Lagrangians $\left\{\mathcal{L}\left(\mathcal{O}(k), h_{k}\right)\right\}_{k=-n-1}^{-1}$ constructed from this collection form a derived Fukaya subcategory equivalent to $D^{b} \operatorname{Coh}\left(\mathbb{P}^{n}\right)$. From now on, we only consider these objects, as well as their cones, shifts and sums in the Fukaya category of $M$. Let us recall some basic facts concerning the geometry of cotangent bundles from [23].

The next lemma shows that $\mathcal{L}\left(\mathcal{O}(-1), h_{-1}\right)$ is a graph over an open set.

Lemma 3.3. Let $\mathcal{T}=\left\{\left(\gamma_{1}, \ldots, \gamma_{n}\right) \mid \gamma_{i}<0\right.$, and $\left.\sum_{i=1}^{n} \gamma_{i}>-1\right\}$, an $n$-cell in the base $\left(S^{1}\right)^{n}$ of $M=T^{*}\left(S^{1}\right)^{n}$. The Lagrangian $\mathcal{L}\left(\mathcal{O}(-1), h_{-1}\right)$ is the graph $\Gamma_{d f}$ of an exact 1 -form $d$ for some $f: \mathcal{T} \rightarrow \mathbb{R}$ (Figure 2 ).

\footnotetext{
${ }^{2}$ This definition differs from the "wrapped" category of Fukaya-Seidel-Smith [13].
} 


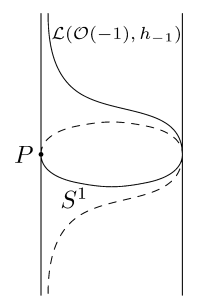

Figure 2: The Landau-Ginzburg mirror of $\mathbb{P}^{1}$. The Lagrangian $\mathcal{L}(\mathcal{O}(-1)$, $\left.h_{-1}\right)$ is a graph over the open interval $\mathcal{T}=S^{1} \backslash P$.

Proof. We know that $\mathcal{L}\left(\mathcal{O}(-1), h_{-1}\right)$ is given by $n$ equations

$$
\gamma_{j}=-\frac{r_{j}^{2}}{1+\sum_{i=1}^{n} r_{i}^{2}}
$$

for $1 \leq j \leq n$ and $\left(r_{1}, \ldots, r_{n}\right) \in\left(\mathbb{R}^{+}\right)^{n}$. Rewriting $r_{i}$ in the form of $\gamma_{j}$, we get

$$
r_{i}=\left(-\frac{\gamma_{j}}{1+\sum_{j=1}^{n} \gamma_{j}}\right)^{1 / 2}
$$

for $1 \leq i \leq n$ and $\left(\gamma_{1}, \ldots, \gamma_{n}\right) \in \mathcal{T}$. Using the coordinate system $(y, \gamma)$ on the cotangent bundle, where $y_{i}=\log r_{i}$, we find

$$
y_{i}=\frac{1}{2} \log \left(-\frac{\gamma_{i}}{1+\sum_{j=1}^{n} \gamma_{j}}\right) \text {. }
$$

Now note

$$
y_{i}=\frac{\partial f}{\partial \gamma_{i}}
$$

where

$$
f=\frac{1}{2} \sum_{i=1}^{n} \gamma_{i} \log \left(-\gamma_{i}\right)-\frac{1}{2}\left(1+\sum_{j=1}^{n} \gamma_{j}\right) \log \left(1+\sum_{j=1}^{n} \gamma_{j}\right)
$$

Lemma 3.4. There are canonical brane structures for objects $\mathcal{L}\left(\mathcal{O}(k), h_{k}\right)$ for $k \in\{-n-1, \ldots,-1\}$. Hence we have obtained $n$ objects $\mathcal{L}\left(\mathcal{O}(k), h_{k}\right)$ in the Fukaya category $\operatorname{Fuk}(M)$. 
Proof. The Lagrangian $\mathcal{L}\left(\mathcal{O}(-1), h_{-1}\right)$ is canonically Hamiltonian isotopic to $\mathcal{T}$, inside $\left.T^{*}\left(S^{1}\right)^{n}\right|_{\mathcal{T}}$ (this is a hamiltonian isotopy inside $\left.T^{*}\left(S^{1}\right)^{n}\right|_{\mathcal{T}}$ but not the whole $\left.T^{*}\left(S^{1}\right)^{n}\right)$. Let $p: T^{*}\left(S^{1}\right)^{n} \rightarrow\left(S^{1}\right)^{n}$ be the projection to the base. This Hamiltonian isotopy can be achieved by the Hamiltonian flow $\varphi_{H, t}$, where $H=f \circ p$, which takes $\mathcal{L}\left(\mathcal{O}(-1), h_{-1}\right)$ to $(1-t) \mathcal{L}\left(\mathcal{O}(-1), h_{-1}\right)$. In particular, when $t=1$, one arrives at $\mathcal{T}$. We can equip $\mathcal{T}$ with grading 0 . Because $\mathcal{T}$ is canonically Hamiltonian isotopic to $\mathcal{L}\left(\mathcal{O}(-1), h_{-1}\right)$, there is a canonical grading for $\mathcal{L}\left(\mathcal{O}(-1), h_{-1}\right)$. As for the pin structure, since $\mathcal{L}\left(\mathcal{O}(-1), h_{-1}\right)$ is contractible, it has a trivial pin structure. So we obtain a canonical brane structure on $\mathcal{L}\left(\mathcal{O}(-1), h_{-1}\right)$.

Let $\widetilde{S}^{1}=\mathbb{R} /(n+1) \mathbb{Z}$, and let $\widetilde{M}=T^{*}\left(\widetilde{S}^{1}\right)^{n}$. Consider the $(n+1)^{n}$ covering $\pi: \widetilde{M} \rightarrow M$, given by $\pi:(y, \tilde{\gamma} \bmod (n+1)) \mapsto(y, \tilde{\gamma} \bmod 1)$. The variables $\tilde{\gamma}=\left(\tilde{\gamma}_{1}, \ldots, \tilde{\gamma}_{n}\right)$ are $n+1$ periodic in each component. To treat $\mathcal{L}\left(\mathcal{O}(k), h_{k}\right)$ for $k \leq-2$, we need to consider the lifts of these Lagrangians under this covering map $\pi$. These Lagrangians become graphs over open sets in $\widetilde{M}$ after the lifting (Figure 3 ).

Let $a=\left(a_{1}, \ldots, a_{n}\right) \in(\mathbb{Z} /(n+1))^{n}$, and assume each $a_{i}$ takes integer value from $-n$ to 0 . We can define open sets

$$
\mathcal{U}(k)^{\left(a_{1}, \ldots, a_{i}\right)}=\left\{\tilde{\gamma} \mid \tilde{\gamma}_{i}<a_{i}, \sum_{j=1}^{n} \tilde{\gamma}_{j}>k+\sum_{j=1}^{n} a_{j}\right\}
$$

for $k \in\{-n-1, \ldots,-1\}$. An exact Lagrangian $\mathcal{L}\left(\mathcal{O}(k), h_{k}\right)$ admits $(n+1)^{n}$ possible lifts. Let $f_{-1}^{0}$ be a real function on $\mathcal{U}^{0}(-1)$, defined by:

$$
f_{-1}^{0}=\frac{1}{2} \sum_{i=1}^{n} \tilde{\gamma}_{i} \log \left(-\tilde{\gamma}_{i}\right)-\frac{1}{2}\left(1+\sum_{j=1}^{n} \tilde{\gamma}_{j}\right) \log \left(1+\sum_{j=1}^{n} \tilde{\gamma}_{j}\right) .
$$

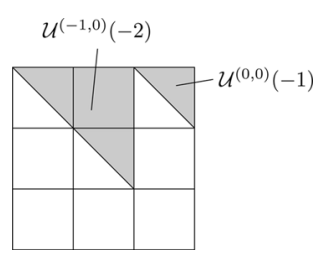

Figure 3: This square represents the base $\left(\widetilde{S}^{1}\right)^{2}$ of $T^{*}\left(\widetilde{S}^{1}\right)^{2}$, as the lift $\widetilde{M}$ of the Landau-Ginzburg mirror $M$ of $\mathbb{P}^{2}$. The Lagrangian $\mathcal{L}^{(0,0)}\left(\mathcal{O}(-1), h_{-1}\right)$ is a graph over the small shaded triangle $\mathcal{U}^{(0,0)}(-1)$, while $\mathcal{L}^{(-1,0)}\left(\mathcal{O}(-2), h_{-2}\right)$ is a graph over $\mathcal{U}^{(-1,0)}(-2)$. 
Note that $f_{-1}^{0}$ is one of the lifts of $f: \mathcal{T} \rightarrow \mathbb{R}$. For $k \in\{-n-1, \ldots,-1\}$, there are $(n+1)^{n}$ lifts of $\mathcal{L}\left(\mathcal{O}(k), h_{k}\right)$, namely, given by the graph of the differential of

$$
f_{k}^{a}=f_{-1}^{0}\left(\frac{\tilde{\gamma}_{1}-a_{1}}{-k}, \frac{\tilde{\gamma}_{2}-a_{2}}{-k}, \ldots, \frac{\tilde{\gamma}_{n}-a_{n}}{-k}\right),
$$

for any $a=\left(a_{1}, \ldots, a_{n}\right) \in(\mathbb{Z} /(n+1))^{n}$. We denote the lifted Lagrangian as the differential of the above function by $\mathcal{L}^{a}\left(\mathcal{O}(k), h_{k}\right)$.

Since the topology of $\mathcal{L}\left(\mathcal{O}(k), h_{k}\right)$ is trivial, it has a trivial (and canonical) pin structure. We know that naturally $\mathcal{L}^{0}\left(\mathcal{O}(k), h_{k}\right)$ has a canonical brane structure, by the same argument for $\mathcal{L}\left(\mathcal{O}(-1), h_{-1}\right)$. The covering map $\pi$ acts trivially on the phase, and hence we can make $\pi$ into a graded covering $\widetilde{\pi}$ with trivial grading. Under the graded covering map $\widetilde{\pi}$, the natural grading of $\mathcal{L}^{0}\left(\mathcal{O}(k), h_{k}\right)$ is mapped to a grading of $\mathcal{L}\left(\mathcal{O}(k), h_{k}\right)$ in $M$, giving a canonical brane structure for $\mathcal{L}\left(\mathcal{O}(k), h_{k}\right)$. Notice that our construction does not depend on the lift of $\mathcal{L}\left(\mathcal{O}(k), h_{k}\right)$. If $\mathcal{L}^{a}\left(\mathcal{O}(k), h_{k}\right)$ is another lift of $\mathcal{L}\left(\mathcal{O}(k), h_{k}\right)$, for any $x \in \mathcal{L}^{0}\left(\mathcal{O}(k), h_{k}\right)$ and $x^{\prime} \in \mathcal{L}^{a}\left(\mathcal{O}(k), h_{k}\right)$ such that $\pi(x)=\pi\left(x^{\prime}\right)$, we have $\widetilde{\alpha}_{\mathcal{L}^{0}\left(\mathcal{O}(k), h_{k}\right)}(x)=\widetilde{\alpha}_{\mathcal{L}^{a}\left(\mathcal{O}(k), h_{k}\right)}\left(x^{\prime}\right)$, where $\widetilde{\alpha}_{\mathcal{L}^{0}\left(k, h_{k}\right)}$ and $\widetilde{\alpha}_{\mathcal{L}^{a}\left(\mathcal{O}(k), h_{k}\right)}$ are canonical gradings of $\mathcal{L}^{0}\left(\mathcal{O}(k), h_{k}\right)$ and $\mathcal{L}^{a}\left(\mathcal{O}(k), h_{k}\right)$, respectively. Hence different lifts give the same grading for $\mathcal{L}\left(\mathcal{O}(k), h_{k}\right)$.

Remark 3.5. Although we are only worrying about finitely many $\mathcal{L}(\mathcal{O}(k)$, $\left.h_{k}\right)$ for $k \in\{-n-1, \ldots,-1\}$, this lemma actually holds for all $k$, i.e., any $\mathcal{L}\left(\mathcal{O}(k), h_{k}\right)$ for $k \in \mathbb{Z}$ has a canonical brane structure and can be made into an object in the Fukaya category $\operatorname{Fuk}(M)$. Therefore, from any line bundle $\mathcal{O}(k)$ on $\mathbb{P}^{n}$, we can construct a Lagrangian brane $\mathcal{L}\left(\mathcal{O}(k), h_{k}\right)$ on the mirror side. Moreover, this construction does not essentially depend on the choice of $T$-invariant metric, although we are using the canonical metric $h_{k}$ here. Different metrics give rise to quasi-isomorphic branes in the Fukaya category. Let $h_{k}^{\prime}=e^{\lambda} h_{k}$ be another metric on $\mathcal{O}(k)$, where $\lambda$ is a $T^{n}$-invariant function on $\mathbb{P}^{n}$. A straightforward calculation shows that the Lagrangian $\mathcal{L}\left(\mathcal{O}(k), h_{k}^{\prime}\right)=\phi_{1}\left(\mathcal{L}\left(\mathcal{O}(k), h_{k}\right)\right.$, where $\phi$ is the hamiltonian flow generated by the function $\lambda$. A more detailed argument of non-characteristic isotopy in [22] shows that $\mathcal{L}\left(\mathcal{O}(k), h_{k}^{\prime}\right)$ and $\mathcal{L}\left(\mathcal{O}(k), h_{k}\right)$ are quasi-isomorphic in the Fukaya category $\operatorname{Fuk}(M)$. For a direct treatment of an arbitrary $T$-invariant metric in the $\mathrm{T}$-duality, please see [11]. In $\mathrm{t}$ ! he rest of this paper, we simply denote $\mathcal{L}\left(\mathcal{O}(k), h_{k}\right)$ by $\mathcal{L}(k)$ for convenience.

When considering homological mirror symmetry, one actually deals with the derived version of the triangulated envelope of the Fukaya category. 
There are several ways to define the triangulated envelope of a Fukaya category. Here we adopt the method of Yoneda embedding, which agrees with the definition in [22].

For any $A_{\infty}$ category $\mathcal{A}$, the Yoneda embedding $\mathcal{Y}: \mathcal{A} \rightarrow \bmod (\mathcal{A})$ maps an object $L \in \mathcal{A}$ to an $A_{\infty}$-module $\operatorname{hom}_{\mathcal{A}}(-, L)$. We write $\operatorname{Tr}(\mathcal{A})$ for the category of twisted complexes of modules in $\mathcal{Y}(\mathcal{A})$ as a version of the triangulated envelope of $\mathcal{A}$.

We study the Fukaya category $\mathcal{F}$ as a full subcategory of $\operatorname{Fuk}(M)$ containing objects $\mathcal{L}(k),-n-1 \leq k \leq-1$, in this paper. Precisely,

Definition 3.6. $\mathcal{F}$ is the full $A_{\infty}$ subcategory of $\operatorname{Fuk}(M)$ consisting of $n$ objects $\mathcal{L}(k)$ where $k \in\{-n-1, \ldots,-1\}$.

The derived category of $\mathcal{F}$, denoted by $D \mathcal{F}$, is a triangulated category $H^{0}(\operatorname{Tr}(\mathcal{F}))$. Note that $D \mathcal{F} \subset D \operatorname{Fuk}(M)$ is a full subcategory. We state our main theorem:

Theorem 3.7. The (bounded) derived Fukaya category $D \mathcal{F}$ is equivalent to $D^{b} \operatorname{Coh}\left(\mathbb{P}^{n}\right)$, the derived category of coherent sheaves on $\mathbb{P}^{n}$.

\subsection{Passing to standard branes}

Let $X$ be a real analytic manifold. A standard brane in the Fukaya category $\operatorname{Fuk}\left(T^{*} X\right)$ over an open set $U \subset X$ is the graph of the differential $d \log m$ equipped with the canonical brane structure, where $m$ is a defining function of $\partial U$ on $\bar{U}: m=0$ on $\partial U$ and $m>0$ on $U$. The quasi-isomorphism class of this brane does not depend on the choice of the particular $m$.

We would like to remind that the Lagrangian $\mathcal{L}(-1)$ is the graph of the exact differential form $d f$ on the open set $\mathcal{T} \subset\left(S^{1}\right)^{n}$. Note that we cannot claim $\mathcal{L}(-1)$ is a standard brane in $\operatorname{Fuk}(M)$ at present, since $e^{f}$ does not go to 0 near the boundary. However, it looks very much like a standard Lagrangian, i.e., the covector $d f$ points inward near the boundary $\partial \mathcal{T}$ and its length is arbitrarily large. This section shows that $\mathcal{L}(-1)$ is indeed isomorphic to a standard brane over the open set $\mathcal{T}$, which allows us to apply the microlocalization functor in [23] to pass into the category of constructible sheaves.

For any real analytic manifold $X$, let $\operatorname{Sh}_{\text {naive }}(X)$ be the triangulated $\mathrm{dg}$ category whose objects are complexes of sheaves with bounded constructible cohomology, and whose morphisms are the usual complexes of morphisms. Then we take $\operatorname{Sh}(X)$ be the dg quotient of $\operatorname{Sh}_{\text {naive }}(X)$ with respect to the subcategory $\mathcal{N}$ of acyclic objects [17]. 
The result of [23] says that there is an $A_{\infty}$ microlocalization functor from $\operatorname{Sh}(X)$ to $\operatorname{Tr} \operatorname{Fuk}\left(T^{*} X\right)$, such that the induced functor for derived categories $D \operatorname{Sh}(X) \rightarrow D \operatorname{Fuk}\left(T^{*} X\right)$ is an embedding. The functor is generated by sending $i_{*} \mathbb{C}_{U}$ for any open set $i: U \hookrightarrow X$ to the standard Lagrangian over $U$. Particularly, for $X=\left(S^{1}\right)^{n}$, we use $\mu$ as the microlocalization functor from $\operatorname{Sh}\left(\left(S^{1}\right)^{n}\right) \rightarrow \operatorname{Tr} \operatorname{Fuk}(M)$ or the derived version $D \operatorname{Sh}\left(\left(S^{1}\right)^{n}\right) \rightarrow D \operatorname{Fuk}(M)$, depending on the context. Similarly, there is a microlocaliztion functor $\widetilde{\mu}: \operatorname{Sh}\left(\left(\widetilde{S}^{1}\right)^{n}\right) \rightarrow \operatorname{Tr} \operatorname{Fuk}(\widetilde{M})$. Due to this fact, we denote the standard brane over $U$ in $\operatorname{Fuk}(M)$ by $\mu\left(i_{*} \mathbb{C}_{U}\right)$, and the standard brane over $\widetilde{U}$ in $\operatorname{Fuk}(\widetilde{M})$ by $\widetilde{\mu}\left(i_{*} \mathbb{C}_{\widetilde{U}}\right)$.

The normalized geodesic flow $\varphi_{t}$ perturbs the objects when defining the morphisms in $\operatorname{Fuk}(M)$. Since we have already chosen a metric on the base $\left(S^{1}\right)^{n}$ of $M=T^{*}\left(S^{1}\right)^{n}$, i.e., $g=d \gamma_{1}^{2}+\cdots+d \gamma_{n}^{2}$, the normalized geodesic flow $\varphi$ is

$$
\varphi_{t}(y, \gamma)=\left(y, \gamma+t \frac{y^{*}}{\|y\|}\right)
$$

where $y^{*} \in T_{\gamma}\left(S^{1}\right)^{n}$ is the dual of $y \in T_{\gamma}^{*}\left(S^{1}\right)^{n}$ with respect to this metric. Note that this flow is only defined on $\left(T^{*}\left(S^{1}\right)^{n}\right)_{0}=\{(y, \gamma) \in M \mid y \neq 0\} \subset M$, i.e., away from the zero section in the cotangent bundle. Let $s$ be an arbitrary point in $\partial \mathcal{T}$, and $L_{\{s\} *}$ be the brane supported on the fiber Lagrangian $T_{s}^{*}\left(S^{1}\right)^{n}$. Define

$$
\begin{aligned}
\mathcal{L}(-1)_{0} & =\mathcal{L}(-1) \cap\left(T^{*}\left(S^{1}\right)^{n}\right)_{0}, \\
\left(L_{\{s\} *}\right)_{0} & =L_{\{s\} *} \cap\left(T^{*}\left(S^{1}\right)^{n}\right)_{0} .
\end{aligned}
$$

Lemma 3.8. There exists a $\delta>0$, such that

$$
0 \leq t_{1} \leq t_{2}<\delta \Rightarrow \varphi_{t_{1}}\left(\left(L_{\{s\} *}\right)_{0}\right) \cap \varphi_{t_{2}}\left(\mathcal{L}(-1)_{0}\right)=\emptyset .
$$

Proof. The Lagrangian $\mathcal{L}(-1)$ is the graph of $d f$ over the open set $\mathcal{T} \subset\left(S^{1}\right)^{n}$. The function $f$ here, as given in Lemma 3.3 , is

$$
f=\frac{1}{2} \sum_{i=1}^{n} \gamma_{i} \log \left(-\gamma_{i}\right)-\frac{1}{2}\left(1+\sum_{j=1}^{n} \gamma_{j}\right) \log \left(1+\sum_{j=1}^{n} \gamma_{j}\right)
$$

Here we assume $\gamma_{i}$ takes value in $(-1,0)$. Denote $Z_{i}=\gamma_{i}$ for $1 \leq i \leq n$, and

$$
Z_{0}=-1-\sum_{j=1}^{n} \gamma_{j}
$$


Hence the function

$$
f=\frac{1}{2} \sum_{i=0}^{n} Z_{i} \log \left(-Z_{i}\right)
$$

while the open set $\mathcal{T}$ is characterized by

$$
\mathcal{T}=\left\{Z_{i}<0 \mid 0 \leq i \leq n\right\}
$$

Since $s \in \partial \mathcal{T}$, there exists a non-empty subset of index $I \subset\{0, \ldots, n\}$, such that $Z_{i}(s)=0$ for $i \in I$ and $Z_{i}(s)<0$ for $i \in\{0, \ldots, n\} \backslash I$. Note that $1 \leq|I| \leq n$ because of the constraint $\sum_{i=0}^{n} Z_{i}=-1$ so that we cannot have $Z_{i}(s)=0$ for all $0 \leq i \leq n$. There is a bound $R>0$ such that $\left|\log \left(-Z_{i}(s)\right)\right|$ $<R / 2$ for all $i \in\{0, \ldots, n\} \backslash I$. Choose a subset $K \subset\{0, \ldots, n\}$ such that $|K|=n$ and $I \subset K$. Therefore, $\left\{Z_{i} \mid i \in K\right\}$ form a coordinate system on $\left(S^{1}\right)^{n}$. Let $i_{0}=\{0, \ldots, n\} \backslash K$ be the single index that is not in $K$. It is easy to see that the metric

$$
g_{K}=\sum_{i \in K} d Z_{i}^{2}
$$

is equivalent to the standard metric

$$
g=d \gamma_{1}^{2}+\cdots+d \gamma_{n}^{2}=\sum_{i=1}^{n} d Z_{i}^{2}
$$

Therefore, there is a bound $Q$ with

$$
\|\cdot\|_{g_{K}} \leq 1 / Q\|\cdot\|_{g}
$$

For any $M>0$, there is a $\delta>0$, such that for any $\gamma \in \mathcal{T}$ with $\operatorname{dist}_{g}(\gamma, s)$ $<\delta, \log \left(-Z_{i}(\gamma)\right)<-M$ for all $i \in I$ and $\left|\log \left(-Z_{i}(\gamma)\right)\right|<R$ for $i \in\{0, \ldots, n\}$ $\backslash I$. We choose a large $M$ such that $\frac{2 n R}{Q^{2}(M-R)}<1$, and a $\delta$ corresponding to this $M$.

For any $(y, \gamma) \in \mathcal{L}(-1)$ and any given $0 \leq t_{1} \leq t_{2}<\delta$, it suffices to show that $\varphi_{t_{1}}(y, s) \neq \varphi_{t_{2}}(y, \gamma)$ to finish the proof. We know that

$$
\varphi_{t_{1}}(y, s)=\left(y, s+t_{1} \frac{y^{*}}{\|y\|_{g}}\right), \quad \text { and } \quad \varphi_{t_{2}}(y, \gamma)=\left(y, \gamma+t_{2} \frac{y^{*}}{\|y\|_{g}}\right) \text {. }
$$

Therefore, $\varphi_{t_{1}}(y, s)=\varphi_{t_{2}}(y, \gamma)$ implies that $v=\left(t_{2}-t_{1}\right) \frac{y^{*}}{\|y\|_{g}}$, where $v=s-$ $\gamma$ is considered as a vector in $T\left(S^{1}\right)^{n}$. Decompose $v=\sum_{i \in K} v_{i} \partial_{Z_{i}}$. When 
$i \in I$

$$
Z_{i}(s)=0, Z_{i}(\gamma)<0 \Rightarrow v_{i}>0
$$

We will show that $v=\left(t_{2}-t_{1}\right) \frac{y^{*}}{\|y\|_{g}}$ is impossible.

Case 1. $\operatorname{dist}_{g}(\gamma, s)<\delta$. We have

$$
\begin{aligned}
y=d f(\gamma) & =\sum_{i \in K} \frac{\partial f}{\partial Z_{i}} d Z_{i} \\
& =\sum_{i \in K} \frac{1}{2}\left(\log \left(-Z_{i}\right)-\log \left(-Z_{i_{0}}\right)\right) d Z_{i}
\end{aligned}
$$

For $i \in I$,

$$
\frac{\partial f}{\partial Z_{i}}<\frac{1}{2}(-M+R)<0
$$

while for $i \in K \backslash I$,

$$
\left|\frac{\partial f}{\partial Z_{i}}\right|<\frac{1}{2} R<R
$$

The length of $y$ satisfies

$$
\|y\|_{g} \geq Q\|y\|_{g_{K}} \geq Q \sqrt{\sum_{i \in I}\left|\frac{\partial f}{\partial Z_{i}}\right|^{2}} \geq Q \sqrt{\frac{1}{4}(M-R)^{2}} \geq \frac{1}{2} Q(M-R) .
$$

Therefore,

$$
\begin{aligned}
\left\langle v, \frac{y}{\|y\|_{g}}\right\rangle & <\sum_{i \in I} v_{i} \frac{-M+R}{Q(M-R)}+\sum_{i \in K \backslash I}\left|v_{i}\right| \frac{2 R}{Q(M-R)} \\
& <\frac{2 R}{Q(M-R)}\|v\|_{g_{K}}|K \backslash I|<\frac{2 n R\|v\|_{g}}{Q^{2}(M-R)} .
\end{aligned}
$$

Since $\frac{2 n R}{Q^{2}(M-R)}<1$, the inner product

$$
\left\langle v, \frac{y^{*}}{\|y\|_{g}}\right\rangle_{g}=\left\langle v, \frac{y}{\|y\|_{g}}\right\rangle<\|v\|_{g}
$$

Note that the dual $y^{*}$ is taken with respect to the standard metric $g$. We know the length of $\frac{y}{\|y\|_{g}}$ is 1 , and this shows that $y^{*}$ is not parallel and in the same direction with $v$. Thus the equality $v=\left(t_{2}-t_{1}\right) \frac{y^{*}}{\|y\|_{g}}$ is impossible. 
Case 2. $\operatorname{dist}_{g}(\gamma, s) \geq \delta$. Therefore, $v=\left(t_{2}-t_{1}\right) \frac{y^{*}}{\|y\|_{g}}$ is impossible to hold since $\|v\|_{g} \geq \delta$ while the length of the right hand side is $t_{2}-t_{1}<\delta$.

Lemma 3.9. The Lagrangian brane $\mathcal{L}(-1)$ is isomorphic to a standard Lagrangian over the open set $\mathcal{T}$ in the category D Fuk $(M)$. Similarly, all $\mathcal{L}^{a}(k)$ are isomorphic to standard Lagrangians over $\mathcal{U}^{a}(k) \subset\left(\widetilde{S}^{1}\right)^{n}$ in $D \operatorname{Fuk}(\widetilde{M})$.

Proof. Since the functions $f$ and $f_{k}^{a}$ are essentially the same, i.e., only differing by scaling of the domain, it suffices to show this lemma only for $\mathcal{L}(-1)$.

To prove that $\mathcal{Y}(\mathcal{L}(-1)) \cong \mathcal{Y}\left(\mu\left(i_{*} \mathbb{C}_{\mathcal{T}}\right)\right)$, we first fix a triangulation $\Lambda$ of the base $\left(S^{1}\right)^{n}$ containing $\mathcal{T}$ and each stratum of its boundary. The technique of [22] exploits the triangulation to resolve the diagonal standard, i.e., the identity functor. What emerges is that the Yoneda module of any object $\mathcal{Y}(L)$ is expressed in terms of (sums and cones of shifts of) Yoneda modules from standards, $\mathcal{Y}\left(\mu\left(i_{*} \mathbb{C}_{T}\right)\right)$, where $T \in \Lambda$. The coefficient of the Yoneda standard module $\mathcal{Y}\left(\mu\left(i_{*} \mathbb{C}_{T}\right)\right)$, takes the form $\operatorname{hom}_{D \operatorname{Fuk}(M)}\left(L_{\{s\} *}, L\right)$, where $s$ is any point in $T$ (contractibility of $T$ means that the choice is irrelevant up to isomorphism) — see Proposition 4.4.1 and Remark 4.5.1 of $[22]$.

Now apply this to $\mathcal{L}(-1)$. Note that $\Lambda$ contains all strata of $\mathcal{T}$ and $\partial \mathcal{T}$. Let $T \neq \mathcal{T}$ and let $s \in T$. Then if $\bar{T} \cap \mathcal{T}=\emptyset$, clearly $\operatorname{hom}_{D \operatorname{Fuk}(M)}\left(L_{\{s\} *}\right.$, $\mathcal{L}(-1))=0$, since $L_{\{s\} *}$ is just the fiber $T_{s}^{*}\left(S^{1}\right)^{n}$. Otherwise, if $T \cap \partial \mathcal{T}$ is non-empty, then Lemma 3.8 ensures us that the hom space $\operatorname{hom}_{D} \operatorname{Fuk}(M)$ $\left(L_{\{s\} *}, \mathcal{L}(-1)\right)=0$. Finally, if $T=\mathcal{T}$, then since $\mathcal{L}(-1)$ is a graph over $T$, the morphism space $\operatorname{hom}_{D \operatorname{Fuk}(M)}\left(L_{\{s\} *}, \mathcal{L}(-1)\right)=\mathbb{C}$. Therefore, $\mathcal{Y}(\mathcal{L}(-1)) \cong \mathcal{Y}\left(\mu\left(i_{*} \mathbb{C}_{\mathcal{T}}\right)\right)$. Note that the result is independent of how $\Lambda$ was chosen.

\section{The quasi-equivalence of the categories}

Recall $\widetilde{M}=T^{*}\left(\widetilde{S}^{1}\right)^{n}$, where $\widetilde{S}^{1}$ is identified with $\mathbb{R} /(n+1) \mathbb{Z}$. The variable $\tilde{\gamma}=\left(\tilde{\gamma}_{1}, \tilde{\gamma}_{2}, \ldots, \tilde{\gamma}_{n}\right)$ on the base is defined $\bmod n+1$, and $y \in T_{\tilde{\gamma}}^{*}\left(\widetilde{S}^{1}\right)^{n} \cong \mathbb{R}^{n}$ is the variable in the fiber. The covering map $\pi: \widetilde{M} \rightarrow M$ is given by $(y, \tilde{\gamma}$ $\bmod (n+1)) \mapsto(y, \tilde{\gamma} \bmod 1)$.

The Fukaya category $\mathcal{F}$ admits a lift $\widetilde{\mathcal{F}}$ through the covering map $\pi$. The objects of $\widetilde{\mathcal{F}}$ are all lifts of each object in $\mathcal{F}$. A lift $\mathcal{L}^{a}(i)$ of $\mathcal{L}(i)$ is the 
graph of a differential 1-form over an open set

$$
\mathcal{U}(i)^{a}=\left\{\tilde{\gamma} \mid \tilde{\gamma}_{j}<a_{j}, \sum_{j} \tilde{\gamma}_{j}>i+\sum_{j} a_{j}\right\}
$$

where $a=\left(a_{1}, \ldots, a_{n}\right), a_{j} \in \mathbb{Z} /(n+1)$. Here we assume $a_{j}$ takes an integer value ranging from $-n$ to 0 . The morphisms of $\widetilde{\mathcal{F}}$ are inherited from $\operatorname{Fuk}(\widetilde{M})$ as

$$
\operatorname{hom}_{\widetilde{\mathcal{F}}}\left(\mathcal{L}^{a}(i), \mathcal{L}^{b}(j)\right)=\operatorname{hom}_{\mathrm{Fuk}(\widetilde{M})}\left(\mathcal{L}^{a}(i), \mathcal{L}^{b}(j)\right)
$$

The composition maps $m_{k}$ are the same as in $\operatorname{Fuk}(\widetilde{M})$. The category $\widetilde{\mathcal{F}}$ is a full $A_{\infty}$-subcategory of $\operatorname{Fuk}(\widetilde{M})$.

Let $\widetilde{\mathcal{D}}$ be the differential graded category containing objects $i_{*} \mathbb{C}_{\mathcal{U}^{a}(i)}$ for $-n-1 \leq i \leq-1$ and $a=\left(a_{1}, \ldots, a_{n}\right) \in(\mathbb{Z} /(n+1))^{n}$. $\widetilde{\mathcal{D}}$ is a full subcategory of $\overline{\operatorname{Sh}}\left(\left(\overline{\widetilde{S}}^{1}\right)^{n}\right)$.

There is a natural $\Gamma=(\mathbb{Z} /(n+1))^{n}$ action on $\widetilde{M}$ given by the deck transformation, i.e., $\alpha \in \Gamma:(y, \tilde{\gamma}) \mapsto(y, \tilde{\gamma}+\alpha)$. This action gives rise to actions on $\widetilde{\mathcal{F}}$ and on $\widetilde{\mathcal{D}}$. For any $\alpha \in \Gamma$, we have for objects

$$
\alpha \cdot \mathcal{L}^{a}(i)=\mathcal{L}^{a+\alpha}(i), \quad \alpha \cdot i_{*} \mathbb{C}_{\mathcal{U}^{a}(i)}=i_{*} \mathbb{C}_{\mathcal{U}^{a+\alpha}(i)}
$$

For morphisms, these actions induce natural isomorphisms on morphism spaces.

$$
\begin{aligned}
\alpha: \operatorname{hom}\left(\mathcal{L}^{a}(i), \mathcal{L}^{b}(j)\right) & \rightarrow \operatorname{hom}\left(\mathcal{L}^{a+\alpha}(i), \mathcal{L}^{b+\alpha}(j)\right) \\
\alpha: \operatorname{hom}\left(i_{*} \mathbb{C}_{\mathcal{U}^{a}(i)}, i_{*} \mathbb{C}_{\mathcal{U}^{b}(j)}\right) & \rightarrow \operatorname{hom}\left(i_{*} \mathbb{C}_{\mathcal{U}^{a+\alpha}(i)}, i_{*} \mathbb{C}_{\mathcal{U}^{b+\alpha}(j)}\right) .
\end{aligned}
$$

The action respects the compositions of corresponding morphisms.

Definition 4.1. The category $\widetilde{\mathcal{F}} / \Gamma$ is the quotient of $\widetilde{\mathcal{F}}$ with respect to the action of $\Gamma$. It consists of $n+1$ objects, denoted as formal orbits $\left(\oplus_{a} \mathcal{L}^{a}(i)\right) / \Gamma$. The morphisms are defined to be

$$
\operatorname{hom}_{\widetilde{\mathcal{F}} / \Gamma}\left(\left(\oplus_{a} \mathcal{L}^{a}(i)\right) / \Gamma,\left(\oplus_{b} \mathcal{L}^{b}(j)\right) / \Gamma\right)=\left(\bigoplus_{a, b} \operatorname{hom}_{\widetilde{\mathcal{F}}}\left(\mathcal{L}^{a}(i), \mathcal{L}^{b}(j)\right)\right) / \Gamma .
$$

The compositions are inherited naturally from $\tilde{\mathcal{F}}$. The quotient category $\widetilde{\mathcal{D}} / \Gamma$ is defined the same way. 
By Lemma 3.9, the branes $\mathcal{L}^{a}(i)$ are quasi-isomorphic to standards over $\mathcal{U}^{a}(i)$. Hence, we have

Proposition 4.2. The $A_{\infty}$ functor $\tilde{\mu}$ restricted on $\mathcal{D}$ gives rise to a quasiequivalence $\tilde{\mu}: \widetilde{\mathcal{D}} \rightarrow \widetilde{\mathcal{F}}$, where $\widetilde{\mu}$ sends $i_{*} \mathbb{C}_{\mathcal{U}^{a}(i)}$ to $\mathcal{L}^{a}(i)$. This functor is a quasi-isomorphism.

Proof. This is essentially the result of [23], and it is obvious that $\widetilde{\mu}$ is T-equivariant.

Taking the quotient of $\widetilde{\mathcal{F}}$ by the action $\Gamma$, we get an $A_{\infty}$ Fukaya-type category $\widetilde{\mathcal{F}} / \Gamma$. This category is isomorphic to $\mathcal{F}$.

Lemma 4.3. $\tilde{\mathcal{F}} / \Gamma \cong \mathcal{F}$.

Proof. Define a functor $J$ sending $\left(\oplus_{a} \mathcal{L}^{a}(i)\right) / \Gamma$ to $\mathcal{L}(i)$. Any morphism from $\left(\oplus_{a} \mathcal{L}^{a}(i)\right) / \Gamma$ to $\left(\oplus_{b} \mathcal{L}^{b}(j)\right) / \Gamma$ corresponds to $(n+1)^{n}$ intersection points (after perturbation) between $\mathcal{L}^{a_{0}+c}(i)$ and $L^{b_{0}+c}(j)$ for some $a_{0}, b_{0}$ and all $c \in \Gamma$. These points are of the same degree $d$. Under the map $\pi$, all these intersection points go to one intersection point between $\mathcal{L}(i)$ and $\mathcal{L}(j)$ of the same degree $d$. On the other hand, any morphism between $\mathcal{L}(i)$ and $\mathcal{L}(j)$ can be lifted to $(n+1)^{n}$ intersection points between $\mathcal{L}^{a_{0}+c}(i)$ and $\mathcal{L}^{b_{0}+c}(j)$ for some $a_{0}, b_{0}$ and all $c \in \Gamma$. Hence the morphism spaces of $\mathcal{F} / \Gamma$ and $\mathcal{F}$ are identical.

For compositions, let us consider a polygon $Q$ bounded by $\mathcal{L}\left(i_{1}\right), \ldots$, $\mathcal{L}\left(i_{k}\right)$. This polygon contributes to the composition of morphisms represented by each vertex. Since $Q$ is a simply-connected polygon, it has $(n+1)^{n}$ lifts to $\widetilde{M}$, the polygons $Q^{c}$ bounded by $\mathcal{L}^{a_{1}+c}\left(i_{1}\right), \ldots, \mathcal{L}^{a_{k}+c}\left(i_{k}\right)$, for some $a_{1}, \ldots, a_{k}$ and all $c \in \Gamma$. Similarly, the compositions in $\widetilde{\mathcal{F}} / \Gamma$ come from counting of the orbit of polygons bounded by $\mathcal{L}^{a_{1}+c}\left(i_{1}\right), \ldots, \mathcal{L}^{a_{k}+c}\left(i_{k}\right)$ for some $a_{1}, \ldots, a_{k}$ and all $c \in \Gamma$. Hence we have proved the composition maps in both $\widetilde{\mathcal{F}} / \Gamma$ and $\mathcal{F}$ are the same.

Since the functor $\widetilde{\mu}$ is $\Gamma$-equivariant, the induced functor $\widetilde{\mathcal{D}} / \Gamma \rightarrow \widetilde{\mathcal{F}} / \Gamma \cong$ $\mathcal{F}$ is a quasi-equivalence. Hence the functor (still denoted by $\widetilde{\mu}$ ) between the derived categories $\widetilde{\mu}: D(\widetilde{\mathcal{D}} / \Gamma) \rightarrow D \mathcal{F}$ is a equivalence.

Lemma 4.4. $D(\widetilde{\mathcal{D}} / \Gamma)$ is equivalent to $D \mathcal{F}$.

The quiver structure of $D(\widetilde{\mathcal{D}} / \Gamma)$ can be computed in a combinatorial way. Note that $D(\widetilde{\mathcal{D}} / \Gamma)$ is generated by objects in $\widetilde{\mathcal{D}} / \Gamma$, as a triangulated 
category. Hence we only deal with objects in $\widetilde{\mathcal{D}}$. The following statement is Lemma 4.4.1. from [23] concerning the morphisms of sheaves.

Lemma 4.5. Let $X$ be a real analytic manifold. For any two open sets $i_{0}: U_{0} \hookrightarrow X, i_{1}: U_{1} \hookrightarrow X$, we have a canonical quasi-isomorphism in the $d g$ category:

$$
\operatorname{hom}_{\mathrm{Sh}(X)}\left(i_{0 *} \mathbb{C}_{U_{0}}, i_{1 *} \mathbb{C}_{U_{1}}\right) \simeq\left(\Omega\left(\bar{U}_{0} \cap U_{1}, \partial U_{0} \cap U_{1}\right), d\right)
$$

The composition of morphisms coincides with the wedge product of differential forms.

By this lemma,

$$
\operatorname{hom}_{\widetilde{\mathcal{D}}}\left(i_{*} \mathbb{C}_{\mathcal{U}^{a}(i)}, i_{*} \mathbb{C}_{\mathcal{U}^{b}(j)}\right) \simeq \Omega\left(\left(\overline{\mathcal{U}^{a}(i)} \cap \mathcal{U}^{b}(j), \partial \mathcal{U}^{a}(i) \cap \mathcal{U}^{b}(j)\right), d\right)
$$

Hence

$$
\operatorname{hom}_{\widetilde{\mathcal{D}} / \Gamma}(\mathcal{U}(i), \mathcal{U}(j))=\left(\bigoplus_{a, b} \Omega\left(\left(\overline{\mathcal{U}^{a}(i)} \cap \mathcal{U}^{b}(j), \partial \mathcal{U}^{a}(i) \cap \mathcal{U}^{b}(j)\right), d\right)\right) / \Gamma
$$

with $\Gamma$ acting on the space of differential forms in the obvious way. Notice that when $\mathcal{U}^{a}(i) \supset \mathcal{U}^{b}(j)$, we have $\Omega\left(\left(\overline{\mathcal{U}^{a}(i)} \cap \mathcal{U}^{b}(j), \partial \mathcal{U}^{a}(i) \cap \mathcal{U}^{b}(j)\right), d\right)=$ $\Omega\left(\mathcal{U}^{b}(j), d\right)$. The cohomology $H^{*}\left(\Omega\left(\mathcal{U}^{b}(j), d\right)\right)=\mathbb{C}[0](\mathbb{C}$ in the zeroeth degree). Otherwise when $\mathcal{U}^{a}(i) \not \supset \mathcal{U}^{b}(j)$,

$$
\begin{aligned}
& \Omega\left(\left(\overline{\mathcal{U}^{a}(i)} \cap \mathcal{U}^{b}(j), \partial \mathcal{U}^{a}(i) \cap \mathcal{U}^{b}(j)\right), d\right) \\
& \left.\quad \cong \Omega\left(\overline{\mathcal{U}^{a}(i)} \cap \mathcal{U}^{b}(j) /\left(\partial \mathcal{U}^{a}(i) \cap \mathcal{U}^{b}(j)\right), p t\right), d\right) .
\end{aligned}
$$

It follows that

$$
\begin{aligned}
& H^{*}\left(\Omega\left(\left(\overline{\mathcal{U}^{a}(i)} \cap \mathcal{U}^{b}(j), \partial \mathcal{U}^{a}(i) \cap \mathcal{U}^{b}(j)\right), d\right)\right) \\
& =H^{*}\left(\overline{\mathcal{U}^{a}(i)} \cap \mathcal{U}^{b}(j) /\left(\partial \mathcal{U}^{a}(i) \cap \mathcal{U}^{b}(j)\right), p t\right) \\
& =0 .
\end{aligned}
$$


This is because when $\mathcal{U}^{a}(i) \not \supset \mathcal{U}^{b}(j), \overline{\mathcal{U}^{a}(i)} \cap \mathcal{U}^{b}(j) /\left(\partial \mathcal{U}^{a}(i) \cap \mathcal{U}^{b}(j)\right)$ is a contractible space, and the homology is zero. Therefore, in the derived category

$$
\begin{aligned}
& \operatorname{hom}_{D(\widetilde{\mathcal{D}} / \Gamma)}(\mathcal{U}(i), \mathcal{U}(j)[k]) \\
& \left.\cong H^{k}\left(\left(\bigoplus_{a, b} \Omega\left(\overline{\mathcal{U}^{a}(i)} \cap \mathcal{U}^{b}(j), \partial \mathcal{U}^{a}(i) \cap \mathcal{U}^{b}(j)\right), d\right)\right) / \Gamma\right) \\
& \left.=\left(\bigoplus_{a, b} H^{k}\left(\Omega\left(\overline{\mathcal{U}^{a}(i)} \cap \mathcal{U}^{b}(j), \partial \mathcal{U}^{a}(i) \cap \mathcal{U}^{b}(j)\right), d\right)\right)\right) / \Gamma \\
& =\bigoplus_{\mathcal{U}^{0}(i) \supset \mathcal{U}^{b}(j)} H^{k}\left(\Omega\left(\mathcal{U}^{b}(j), d\right)\right) \\
& \cong \begin{cases}\mathbb{C}^{N_{n}(i-j)}, & k=0, \\
0, & k \neq 0 .\end{cases}
\end{aligned}
$$

Here $N_{n}(i-j)=\#\left\{b=\left(b_{1}, \ldots, b_{n}\right) \mid b_{i} \leq 0, \sum b_{i} \geq i-j\right\}$, counting all possible $\mathcal{U}^{b}(j)$ in $\mathcal{U}^{0}(i)$. Notice this explicit calculation of morphisms implies that $\{\mathcal{U}(-n-1), \ldots, \mathcal{U}(-1)\}$ is a full strong exceptional collection of $D(\widetilde{\mathcal{D}}$ $/ \Gamma)$, since $N_{n}(0)=1$ and $N_{n}(m)=0$ when $m<0$. Let $e_{i, j}^{b}$ denote the identity in $H^{0}\left(\Omega\left(\mathcal{U}^{b}(j), d\right)\right.$ as a subspace of $\operatorname{hom}_{D(\widetilde{\mathcal{D}} / \Gamma)}(\mathcal{U}(i), \mathcal{U}(j))$.

We compare this exceptional collection with the exceptional collection $\{\mathcal{O}(-n-1), \ldots, \mathcal{O}(-1)\}$ of $D^{b} \operatorname{Coh}\left(\mathbb{P}^{n}\right)$. The morphism spaces of $D(\widetilde{\mathcal{D}} / \Gamma)$ are

$$
\operatorname{hom}_{D(\widetilde{\mathcal{D}} / \Gamma)}(\mathcal{U}(i), \mathcal{U}(j))=\bigoplus_{\mathcal{U}^{0}(i) \supset \mathcal{U}^{b}(j)} H^{0}\left(\Omega\left(\mathcal{U}^{b}(j), d\right)\right) .
$$

The constraint on the multi-index $b$ is that

$$
b=\left(b_{1}, \ldots b_{n}\right) \in\left\{b=\left(b_{1}, \ldots, b_{n}\right) \mid b_{i} \leq 0, \sum b_{i} \geq i-j\right\} .
$$

We construct an isomorphism $\nu$ of morphisms spaces $\operatorname{hom}_{D(\widetilde{\mathcal{D}} / \Gamma)}(\mathcal{U}(i), \mathcal{U}(j))$ to $\operatorname{hom}_{D^{b} \operatorname{Coh}\left(\mathbb{P}^{n}\right)}(\mathcal{O}(i), \mathcal{O}(j))$ by

$$
\begin{array}{ccc}
\nu: \operatorname{hom}_{D(\widetilde{\mathcal{D}} / \Gamma)}(\mathcal{U}(i), \mathcal{U}(j)) & \cong & \operatorname{hom}_{D^{b} \operatorname{Coh}\left(\mathbb{P}^{n}\right)}(\mathcal{O}(i), \mathcal{O}(j)) \\
e_{i, j}^{b} & \mapsto & x_{i, j}^{b}
\end{array}
$$

where $x_{i, j}^{b}=x_{0}^{j-i+\sum b_{i}} x_{1}^{-b_{1}} \cdots x_{n}^{-b_{n}}$ is a generator of $\operatorname{hom}_{D^{b} \operatorname{Coh}\left(\mathbb{P}^{n}\right)}(\mathcal{O}(i)$, $\mathcal{O}(j))$ (Figure 4). 


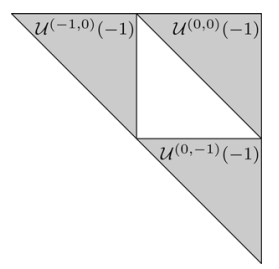

Figure 4: The case of $\mathbb{P}^{2}$. On $\left(\widetilde{S}^{1}\right)^{2}$, the base of $\widetilde{M}, \mathcal{U}^{(0,0)}(-1), \mathcal{U}^{(0,1)}(-1)$ and $\mathcal{U}^{(1,0)}(-1)$ (three shaded triangles) inside $\mathcal{U}^{(0,0)}(-2)$ (the larger triangle) correspond to three generators of $\operatorname{hom}_{D^{b} \operatorname{Coh}\left(\mathbb{P}^{2}\right)}(\mathcal{O}(-2), \mathcal{O}(-1))$.

The next step is to show that this identification of morphisms respect composition maps. For $i<j<k$, morphism spaces $\operatorname{hom}_{D(\widetilde{\mathcal{D}} / \Gamma)}(\mathcal{U}(i), \mathcal{U}(j))$ and $\operatorname{hom}_{D(\widetilde{\mathcal{D}} / \Gamma)}(\mathcal{U}(j), \mathcal{U}(k))$ are non-trivial. The composition map is computed as wedge product of cohomology in the following decomposition.

$$
\begin{aligned}
& \operatorname{hom}_{D(\widetilde{\mathcal{D}} / \Gamma)}(\mathcal{U}(j), \mathcal{U}(k)) \otimes \operatorname{hom}_{D(\widetilde{\mathcal{D}} / \Gamma)}(\mathcal{U}(i), \mathcal{U}(j)) \\
& =\bigoplus_{\mathcal{U}^{b}(j) \subset \mathcal{U}^{0}(i)} \bigoplus_{\mathcal{U}^{b+c}(k) \subset \mathcal{U}^{b}(j)} H^{0}\left(\Omega\left(\overline{\mathcal{U}^{b+c}(k)}, d\right)\right) \otimes H^{0}\left(\Omega\left(\overline{\mathcal{U}^{b}(j)}, d\right)\right) .
\end{aligned}
$$

Therefore, one observes that the multiplication restricted on subspaces of subspaces $H^{0}\left(\Omega\left(\overline{\mathcal{U}^{b+c}(k)}, d\right)\right)$ and $H^{0}\left(\Omega\left(\overline{\mathcal{U}^{b+c}(k)}, d\right)\right)$ gives rise to $e_{j, k}^{c} \cdot e_{i, j}^{b}=$ $e_{i, k}^{b+c}$.

Under the isomorphism $\nu, e_{j, k}^{c}$ and $e_{i, j}^{b}$ correspond to $x_{j, k}^{c}$ and $x_{i, j}^{b}$, respectively. The composition

$$
\begin{aligned}
x_{j, k}^{c} \cdot x_{i, j}^{b} & =x_{0}^{k-j+\sum c_{i}} x_{1}^{-c_{1}} \cdots x_{n}^{-c_{n}} \cdot x_{0}^{j-i+\sum b_{i}} x_{1}^{-b_{1}} \cdots x_{n}^{-b_{n}} \\
& =x_{0}^{k-i+\sum\left(b_{i}+c_{i}\right)} x_{1}^{-b_{1}-c_{1}} \cdots x_{n}^{-b_{n}-c_{n}} \\
& =x_{i, k}^{b+c}
\end{aligned}
$$

which means $\nu\left(e_{j, k}^{c} \cdot e_{i, j}^{b}\right)=x_{j, k}^{c} \cdot x_{i, j}^{b}$. Therefore, we have shown the quiver structures of two full strong exceptional collections are the same, the equivalence of triangulated categories $D^{b} \operatorname{Coh}\left(\mathbb{P}^{n}\right) \cong D(\widetilde{\mathcal{D}} / \Gamma)$ follows. By Lemma 4.4, our main theorem holds.

Theorem 4.6. $D \mathcal{F}$ is equivalent to $D^{b} \operatorname{Coh}\left(\mathbb{P}^{n}\right)$.

Remark 4.7. The Lagrangians $\mathcal{L}^{a}(i)$ for $i \in\{-n-1, \ldots,-1\}$ are standard branes over particular open sets (after the lift with respect to the covering map $\pi$ ). They correspond to certain constructible sheaves with 
respect to a fixed stratification $\widetilde{\Lambda}$. Pushing forward to the constructible sheaves on $\left(S^{1}\right)^{n}$ via the covering map $\pi$, they are constructible sheaves on $\left(S^{1}\right)^{n}$ with respect to the stratification $\Lambda$, which coincides with the stratification given by Bondal [7]. Bondal arrives at this stratification from the coherent sheaves on a toric variety, while we obtain this stratification via the standard Lagrangians constructed by T-duality.

\section{References}

[1] M. Abouzaid, Homogeneous coordinate rings and mirror symmetry for toric varieties, Geom. Topol. 10 (2006), 1097-1157, math.SG/0511644.

[2] — Morse homology, tropical geometry, and homological mirror symmetry for toric varieties, math.SG/0610004.

[3] D. Arinkin and A. Polishchuk, Fukaya category and Fourier transform, Winter School on Mirror Symmetry, Vector Bundles and Lagrangian Submanifolds (Cambridge, MA, 1999), AMS/IP Stud. Adv. Math., 23, Amer. Math. Soc., Providence, RI, 2001, 261-274, math/9811023.

[4] D. Auroux, Mirror symmetry and T-duality in the complement of an anticanonical divisor, arXiv: 0706.3207.

[5] D. Auroux, L. Katzarkov and D. Orlov, Mirror symmetry for weighted projective planes and their noncommutative deformations, math.AG/0404281.

[6] —-, Mirror symmetry for Del Pezzo surfaces: vanishing cycles and coherent sheaves, Invent. Math. 166 (2006), 537-582, math.AG/0506166.

[7] A. Bondal, Integrable systems related to triangulated categories, talk at MSRI workshop on Generalized McKay Correspondences and Representation Theory, regarding the joint work with W.-D. Ruan, March 21, 2006, (video is at www.msri.org/ communications/vmath/VMathVideos/VideoInfo/2469/show video).

[8] - Derived categories of toric varieties, Convex and Algebraic Geometry, Oberwolfach Conference Reports, EMS Publishing House $3,2006,284-286$. 
[9] K. Chan and N.C. Leung, Mirror symmetry for toric fano manifolds via SYZ transformations, arXiv: 0801:2830.

[10] C.-H. Cho and Y.-G. Oh, Floer cohomology and disc instantons of Lagrangian torus fibers in Fano toric manifolds, Asian J. Math. 10 (2006), 773-814, math.SG/0308225.

[11] B. Fang, C-C. Liu, D. Treumann, E. Zaslow, T-duality and equivariant homological mirror symmetry for toric varieties, arXiv: 0811.1228.

[12] K. Fukaya, Y.-G. Oh, H. Ohta and K. Ono, Lagrangian intersection Floer theory: anomaly and obstruction, preprint, second expanded version, 2006.

[13] K. Fukaya, P. Seidel and I. Smith, Exact Lagrangian submanifolds in simply-connected cotangent bundles, math/0701783.

[14] K. Hori, Mirror symmetry and quantum geometry, Proceedings of the International Congress of Mathematicians, Vol. III (Beijing, 2002), Higher Ed. Press, Beijing, 2002, 431-443, hep-th/0207068.

[15] K. Hori, A. Iqbal and C. Vafa, D-branes and mirror symmetry, hepth/0005247.

[16] K. Hori and C. Vafa, Mirror symmetry, hep-th/0002222.

[17] B. Keller, On the cyclic homology of exact categories, J. Pure Appl. Algebra 136(1) (1999), 1-56.

[18] M. Kontsevich, Lectures at ENS, Paris, Spring 1998, notes taken by J. Bellaiche, J.-F. Dat, I. Marin, G. Racinet and H. Randriambololona.

[19] - Homological algebra of mirror symmetry, Proc. International Congress of Mathematicians (Zürich, 1994), Birkhäuser, Basel, 1995, $120-139$.

[20] N.C. Leung and C. Vafa, Branes and toric geometry, Adv. Theor. Math. Phys. 2(1) (1998), 91-118.

[21] N.C. Leung, S.-T. Yau and E. Zaslow, From special Lagrangian to Hermitian-Yang-Mills via Fourier-Mukai Transform, Adv. Theor. Math. Phys. 4(6) (2000), 1319-1341.

[22] D. Nadler, Microlocal branes are constructible sheaves, math/0612399.

[23] D. Nadler and E. Zaslow, Constructible sheaves and the Fukaya category, math/0604379. 
[24] A. Strominger, S.-T. Yau and E. Zaslow, Mirror symmetry is T-duality, Nucl. Phys. B 479 (1996), 243-259, hep-th/9606040.

Department of Mathematics

NorthWESTERn University

2033 SHERIDAN ROAD

EVANSTON

IL 60208

USA

E-mail address: b-fang@math.northwestern.edu

Received September 13, 2008 